\title{
Effects of $\beta$-glucan on some environmental toxins: An overview
}

\author{
Vaclav Vetvicka
}

Background. Beta-glucans are naturally occurring polysaccharides and constituents of the cell wall of certain pathogenic bacteria and fungi. They have proven healing and immunostimulating properties, linked to enhanced macrophage and natural killer cell function which likely involves specific interaction with several cell surface receptors, such as lactosylceramide, selected scavenger receptors, and dectin-1 (betaGR). In particular, glucan reduces the immunosuppressive effects of a number of agents including chemo therapy and radiation. More recent studies suggest a positive function for glucan in the immunosuppression caused by toxic agents in the environment.

Aim. An overview of the effects of glucan on the mycotoxin, aflotoxin and other environmental toxins (mercurythimerosal, depleted uranium).

Conclusion. Glucan is effective as a natural immunomodulator and could be used as an inexpensive solution to reducing the adverse effects of some environmental toxins.

Key words: beta-glucan, aflotoxin, mercury, depleted uranium, detoxification, immunity

Received: August 30, 2013; Accepted with revision: November 22, 2013; Available online: December 11, 2013 http://dx.doi.org/10.5507/bp.2013.090

Department of Pathology, University of Louisville, 511 S. Floyd, MDR Bldg., Rm. 224, Louisville, KY 40292, USA Corresponding author: Vaclav Vetvicka, e-mail: vaclav.vetvicka@louisville.edu

\section{INTRODUCTION}

Environmental toxins have significant adverse effects on the health of both animals and man. Such toxins increase host susceptibility to infections, compromise the immune system and increase the risk of autoimmune disease. These issues have led to large epidemiological studies that attempt to establish sets of immunologically relevant end points ${ }^{1}$.

Glucans are a major constituent of the cell wall of certain saprophytic and pathogenic fungi. For many years, they have been shown to reduce the immunosuppressive effects of a number of factors, including chemo and radiation treatment ${ }^{2-5}$. Following discovery of the effects of glucan on bone marrow protection and activation of bone marrow progenitor cells, studies have suggested new functions for glucan. These are based on the well-known fact that glucan strongly stimulates all facets of the immune system, from nonspecific response to both branches of the specific immune system ${ }^{6}$. From this, emerged the hypothesis that glucan might offset or at least reduce immunosuppression caused by toxic agents.

\section{GLUCAN AND MYCOTOXINS}

Contamination of the environment from the air to the soil, represents a serious danger not only to commercially farmed animals but also to humans. Some of the most deadly and persistent contaminants of animal feed are aflatoxins. These are naturally occurring mycotoxins that are produced by many species of Aspergillus, predominantly Aspergillus flavus and Aspergillus parasiticus.
High-level aflatoxin exposure causes acute hepatic necrosis that may later result in cirrhosis or carcinoma of the liver. Acute hepatic failure manifests as hemorrhage, edema, alteration in digestion and changes to the absorption and metabolism of nutrients ${ }^{7,8}$. No animal species is immune to the acute toxic effects of aflatoxins and although humans are more resistant, the current data support the danger of hepatocellular cancer in $\operatorname{man}^{9}$.

The levels of aflatoxins in agriculture are controlled by a variety of strategies including use of fungicides and pesticides but these treatments have a significant negative impact on the environment and often incur similar health problems to aflatoxins themselves.

The first studies showed that (1-3)- $\beta$-D-glucan, particularly with the (1-6)- $\beta$-D-glucan side chains, can regulate the presence of aflatoxins. Subsequent research revealed that under physiological conditions, mostly temperature, glucan can absorb up to $50 \%$ of zearalenone molecules $^{10}$, most probably via hydrogen bonding between the hydroxyl, lactone, and ketone groups of the zearalenone molecule with the glucan single helix and van der Waals interactions between the phenyl and the $\beta$-Dglucopyranose moieties.

Further studies focused on interactions between glucan and aflatoxin B1. These showed that glucan was involved in binding to aflotoxin B1. Using molecular methods, it was revealed that hydroxyl, lactone and ketone groups participated in formation of hydrogen bonds and van der Walls interactions between glucan and aflatoxin B1 (ref. ${ }^{11}$ ). The first investigations of glucan as a decontaminant used insoluble glucans isolated from Saccharomyces cerevisiae ${ }^{12}$. A later publication showed that glucan was chemically modified to form crosslinked 
carboxymethyl glucan. The resulting glucan had greatly improved adsorption of the mycotoxin T-2 and zearalenome ${ }^{13}$.

However, to further establish the exact mechanisms of the adsorption of these mycotoxins on glucans, more research will be needed, in particular using a large number of glucan derivates. The exact molecular structure of tetraalkylammonium-modified carboxymethylglucan remains to be determined too.

Another interesting study used the known fact that manno-oligosaccharides can attenuate aflatoxicosis ${ }^{14}$ and that glucans protects DNA against oxidative stress-related damage ${ }^{15}$. In testing the mechanisms involved in the aflatoxin-inhibiting effects of Lentinula edodes cultures, the authors demonstrated that these cultures act as an external stimulus affecting the anti-oxidant status in the fungal cells, which subsequently inhibits aflatoxin. Glucan present in cultures activates the transcription factors related to the anti-oxidant response ${ }^{16}$. Based on this study, glucan acts not „only“ by absorption of mycotoxins but directly reduces aflatoxin production.

When we understand that mushrooms such as $L$. edodes can grow directly on waste materials such as olive mill wastewaters ${ }^{17}$, it is clear how significant this finding might be. After all, Lentinan isolated from this mushroom has been used for treating gastric cancer for more than 30 years ${ }^{18}$. In addition, L. edodes-based glucans have extremely low cellular toxicity. For this reason they can be used directly in animal feed, simultaneously strengthening the immune system of animals and detoxificating their feed ${ }^{19}$.

\section{GLUCAN AND OTHER TOXINS-MERCURY, PERLUOROCTANIC ACID, DEPLETED URANIUM}

Glucan has been found to protect bone marrow against the toxic effects of both chemotherapy and irradiation $^{20,21}$, most probably via direct stimulation of bone marrow progenitor cells. It is not surprising that glucan is attracting interest as a supplement for reducing the sideeffects of cancer treatments. Additional effects of glucan were found using a model of $2.45 \mathrm{Ghz}$ electromagnetic radiation and oxidative injury of the skin. This experiment showed that the prophylactic use of glucan reversed the radiation-caused changes in MDA levels and SOD activities. The authors hypothesized that glucan can offer protection against oxidative injury induced by electromagnetic radiation, most likely through its known anti-oxidant abilities $^{22,23}$.

Our laboratory focused on the ability of yeast-derived insoluble glucan \#300 to decrease the immunosuppressive actions of various immunotoxins including mercury and perluorooctanic acid. The presence of mercury salts in the environment represents a serious problem, resulting in accumulation of mercury in fish ${ }^{24}$ and despite the fact that organic ethylmercury present in limited amounts in vaccines was apparently officially cleared from causing autism ${ }^{25}$, it can still have strong immunosuppressive properties $^{26}$ including lowering of cellular and humoral reactions such as phagocytosis, IL-6 and IL-12 secretion

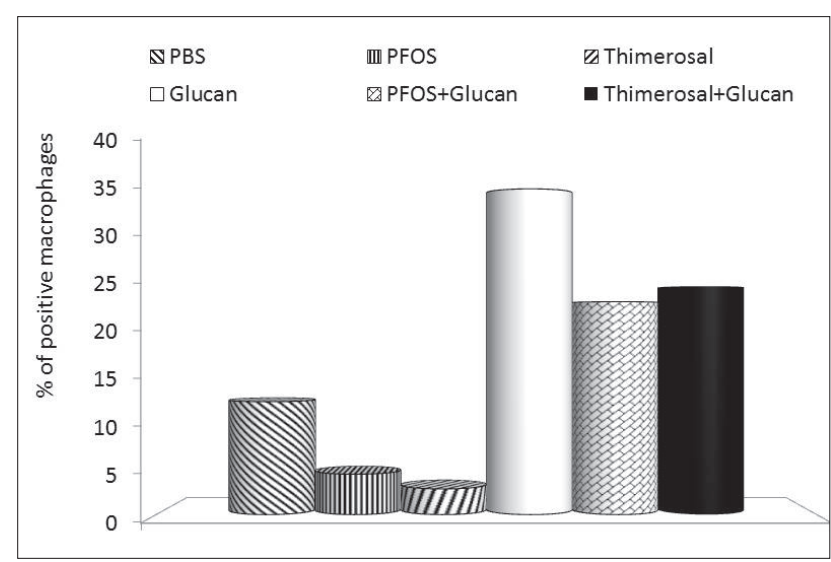

Fig. 1. Effects of orally-administered glucan on reduction of phagocytosis of peritoneal macrophages by thimerosal or perfluooctane sulphuric acid.

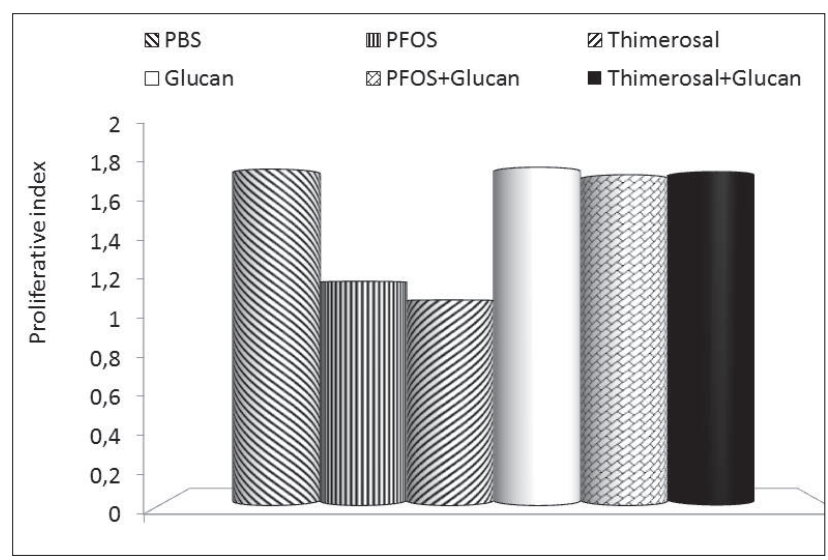

Fig. 2. Effects of orally-administered glucan on reduction of proliferation of spleen-derived T lymphocytes by thimerosal or perfluooctane sulphuric acid.

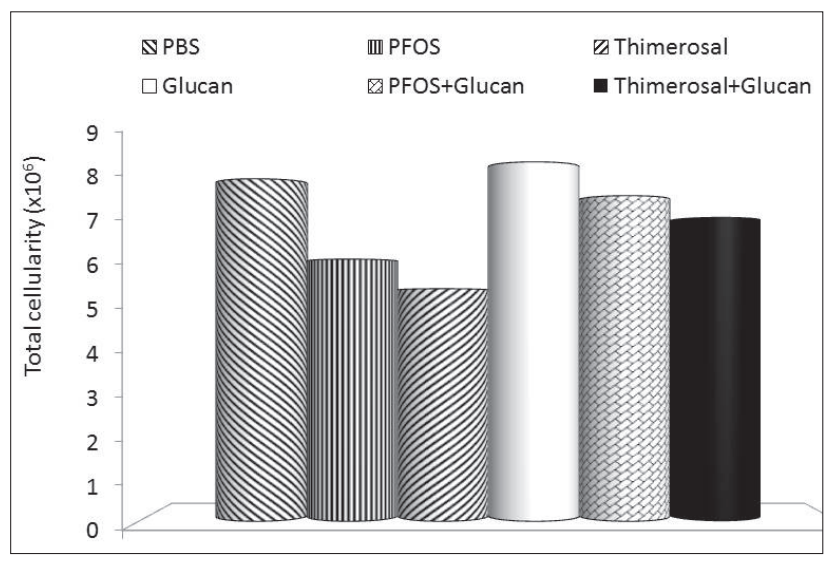

Fig. 3. Effects of orally-administered glucan on reduction of cellularity in spleen by thimerosal or perfluooctane sulphuric acid.

and NK cell activity. Similarly, perfluorooctanesulfonates were found to decrease humoral immunity ${ }^{27}$, IL-6 and IL10 secretion $^{28}$ and natural killer cell activity ${ }^{29}$.

Our studies showed that two-week oral administration of glucan greatly ameliorated the immunosuppressive action of mercury, including IL-6 and IL-12 production, 
Table 1. Antitoxic activity of glucan in vitro and in vivo.

\begin{tabular}{lllc}
\hline Type of glucan & Source & Effects on & Reference \\
\hline Insoluble & Saccharomyces cerevisiae & Adsorption, animal protection & 10 \\
Insoluble & Saccharomyces cerevisiae & Myxotoxin complexation & 11 \\
Alkali-soluble & Saccharomyces cerevisiae & Myxotoxin complexation & 11 \\
Insoluble & Saccharomyces cerevisiae & Adsorption of aflatoxin & 12 \\
Soluble & Saccharomyces cerevisiae & Adsorption of zearalenone and T-2 toxin & 13 \\
Insoluble & Saccharomyces cerevisiae & Oxidative DNA damage & 15 \\
Soluble & Lentinula edodes & Antioxidant enzymes stimulation & 16 \\
Insoluble & Saccharomyces cerevisiae & Antioxidant enzymes & 22 \\
Insoluble & Saccharomyces cerevisiae & IL-6, IL-12, phagocytosis, antibodies & 30 \\
Insoluble & Saccharomyces cerevisiae & Proliferation, NK cell activity & 31 \\
Insoluble & Saccharomyces cerevisiae & Cellularity, proliferation, antibodies & 32 \\
Insoluble & Saccharomyces cerevisiae & Amikain-caused ototoxicity & 33 \\
Insoluble & Saccharomyces cerevisiae & ROS formation, GSH oxidation & 35 \\
\hline
\end{tabular}

antibody secretion, NK cell activity and phagocytosis of peripheral blood cells ${ }^{30}$. Subsequent evaluations revealed that 7 day exposure to mercury caused a significant drop in cellularity in spleen, which was fully restored by glucan supplementation (Fig. 1). Similarly, the suppressed proliferation of $\mathrm{T}$ lymphocytes was returned to normal by feeding with glucan (Fig. 2). The older data on phagocytosis were confirmed using peritoneal macrophages. The stimulation of phagocytic activity caused by glucan was strong enough not only to return the inhibited activity to normal values but to a level that was significantly higher than the phagocytosis found in PBS-treated animals (Fig. 3).

The situation in perfluorooctanesulfonate-induced immunotoxicity closely resembled the toxicity of mercury. In all three tested immune reaction (proliferation of lymphocytes, phagocytic activity, and spleen cellularity), short-term exposure to perfluorooctanesulfonate caused significant reduction, whereas 2 weeks of food supplementation with glucan either returned the reaction to normal values or elevated it to near the level of glucan alone (Fig. 1-3). Previously, we showed that some of the suppression caused by perfluorooctanesulfonates can be decreased or even reversed by a glucan-resveratrol-vitamin $\mathrm{C}$ combination $^{31,32}$, but glucan alone clearly possesses similar effects.

There are additional reports describing the protective effects of glucan against toxicity. One is a report evaluating the effect of glucan on hearing loss caused by amikacin. Amikacin treatment showed significant ototoxicity by causing serious deterioration of hearing at most tested frequencies. Oral administration of glucan together with amikacin limited the hearing loss ${ }^{33}$. In addition, glucan also showed some hearing improvement when used alone. The exact mechanisms are not known but the authors speculate that inhibition of free radical formation might be involved.
Glucan was also tested in the case of depleted uranium toxicity. The toxic effects of this compound manifests mostly via mitochondrial dysfunction, with the most relevant toxic mechanisms believed to be oxidative stress and reactive oxygen species ${ }^{34}$. A detailed study showed that glucan attenuated the formation of depleted uraniuminduced mitochondrial reactive oxygen species, lipid peroxidation and glutathione oxidation. In addition, further mitochondrial dysfunction including outer membrane damage and release of cytochrome $\mathrm{c}$ was prevented ${ }^{35}$.

The mechanisms by which glucan supplementation blocks or at least lowers immunosuppression are currently unclear. One option is simple stimulation of immune reactions which supplements the lowering of immunity caused by exposure to toxins. A second option might be upregulation $^{36}$ or down-regulation ${ }^{37}$ of some important genes, such as ERCC5, CASP9, CYPIAI, CDC42, OKC, BCl-2 or $\mathrm{NF}-\kappa \mathrm{B}$. Clearly, more research is needed before glucan can be fully established in the treatment immunotoxic disorders. The relevant activity of glucan in vitro and in vivo is summarized in Table 1.

\section{CONFLICT OF INTEREST STATEMENT}

The author stated that there are no conflicts of interest regarding the publication of this article.

\section{REFERENCES}

1. Tryphonas H. Approaches to detecting immunotoxic effects of environmental contaminants in humans. Environ Health Perspect 2001;109:877-84.

2. Akimoto M, Ueki H, Nakajima Y, Matano S, Ewasaki H, Abe R, Kasai $\mathrm{M}$. Prevention of 5-FU induced toxicity in $\mathrm{C} 3 \mathrm{H} / \mathrm{HE}$ mice with inter- 
feron or with interferon inducers (polyu 1:C, OK-432, Lentinan). Jpn J Cancer Chemother 1984;11:1462-7.

3. Patchen ML, MacVittie TJ, Brook I. Glucan-induced hemopoietic and immune stimulation: therapeutic effects in sublethally and lethally irradiated mice. Meth Find Exp Clin Pharmacol 1986;8:151-5.

4. Matsuo T, Kurahashio Y, Nishida S, Kumada K, Hayami T, Takagi T. Granulopoietic effects of lentinan in mice: Effects on GM-CFC and 5-FU-induced leukopenia. Jpn J Cancer Chemother 1987;14:1310-4.

5. Hofer M. Pospisil M. Viklicka S. Vacek A. Pipalova I. Bartonickova A Hematopoietic recovery in repeatedly irradiated mice can be enhanced by a repeatedly administered combination of diclofenac and glucan. $\mathrm{J}$ Leukocyte Biol 1993;53:185-9.

6. Vetvicka V, Novak M. (Eds.) Biology and Chemistry of Beta Glucan, Vol. 1, Bentham Science Publ. 2011.

7. Liu U, Chang CCH, Marsh GM, Wu F. Population attributable risk of aflatoxin-related liver cancer: Systematic review and meta-analysis. Eur J Cancer 2012;48:2125-36.

8. Khlangwiset P, Shephard GS, Wu F. Aflatoxins and growth impairment. A review. Crit Rev Toxicol 2011;41:740-55.

9. Matsuda Y, Wakai, T, Kubota M, Osawa M, Sanpei A, Fujimaki S. Mycotoxins are conventional and novel risk biomarkers for hepatocellular carcinoma. World J Gastroenterol 2013;19:2587-90.

10. Yiannikouris A, Francois J, Poughon L, Dussap CG, Bertin G, Jeminet G, Jouany JP. Adsorption of zearalenone by beta-D-glucans in the Saccharomyces cerevisiae cell wall. J Food Prot 2004;67:1195-200.

11. Yiannikouris A, Andre G, Poughon L, Francois J, Dussap CG, Jeminet G, Berin G, Jouany JP. Chemical and conformational study of the interactions involved in mycotoxin complexation with $\beta$-D-glucans. Biomacromolecules 2006; 7:1147-55.

12. Pereyra CM, Cavaglieri LR, Chiacciera SM, Dalcero A. The corn influence on the adsorption levels of aflatoxin $B_{1}$, and zearalenone by yeast cell wall. J Appl Microbiol 2012;114:655-62.

13. Freimund, S, Sauter M, Rys P. Efficient adsorption of the mycotoxins zearalenone and T-2 toxin on a modified yeast glucan. J Environ Sci Health Part B - Pesticides, Food Contaminants and Agricultural Wastes 2003;B38:243-55.

14. Baptista AS, Horii J, Calori-Domingues MA, Ricotti E, Mastrodi Salgao J, Vizioli MR. The capacity of manno-oligosaccharides, thermolysed yeast and active yeast to atenuate aflatoxicosis. J. World Microb Biotechnol 2004;20:475-81.

15. Slamenova D, Labaj J, Krizkova L, Kogan G, Sandula J, Bresgen N, Eckl P. Protective effects of fungal (1-3)- $\beta$ D-glucan derivates against oxidative DNA lesions in V79 hamster lung cells. Cancer Lett 2003;198:153-60.

16. Reverberi M, Fabbri AA, Zjalic S, Ricelli A, Puncelli F, Fanelli C. Antioxidant enzymes stimulation in Aspergillus parasiticus by Lentinula edodes inhibits aflatoxin production. Appl Microbiol Biotechnol 2005;69:207-15.

17. Zjalic S, Fabbri AA, Ricelli A, Reverberi M, Galli E, Fanelli C. Effect of olive oil mill waste waters on the edible medicinal mushroom Lentinus edodes (Berk.). Sing. growth and lignin degrading enzymes. Int J Med Mushrooms 2002;4:85-93.

18. Ina K, Kataoka T, Ando T. The use of lentinan for treating gastric cancer. Anti-Cancer Agents Med Chem 2013;13:681-8.

19. Guo FC, Kwakkel RP, Williams BA, Li WK, Li HS, Luo JHY, Li XP, Wei YX, Yan ZT, Vestegen MW. Effects of mushroom and herb polysaccharides, as alternatives for an antibiotic, on growth performance of broilers. Br Pollut Sci 2004;45:684-94.

20. Patchen ML, MacVittie TJ. Use of glucan to enhance hemopoietic recovery after exposure to cobalt-60 irradiation. Adv Exp Med Biol 1982;155:267-72.

21. Vetvicka V, Dvorak B, Vetvickova J, Richter J, Krizan J, Sima P, Yvin JC. Orally administered marine (1-3)- $\beta$-D-glucan Phycarine stimulates both humoral and cellular immunity. Int J Biol Macromol 2006;40:2918 .

22. Ceyhan AM, Akkaya VB, Gulecol SC, Ceyhan BM, Ozguner F, Chen WC. Protective effects of $\beta$ glucan against oxidative injury induced by 2.45-GHz electromagnetic radiation in the skin tissue of rats. Arch Dermatol Res 2012;304:521-7.

23. Kayali, H, Ozdag MF, Kahraman S, Aydin A, Gonul E, Sayal A, Odobasi Z, Timurkyanak E. The antioxidant effect of $\beta$ glucan on oxidative stress status in experimental spinal cord injury in rats. Neurosurg Rev 2005;28:298-302.

24. Sagiv SK, Thurston SW, Bellinger DC, Amarasiriwardena C, Korrick SA. Prenatal exposure to mercury and fish consumption during pregnancy and attention-deficit/hyperactivity disorder-related behavior in children. Arch Pediatr Adolesc Med 2012;166:1123-31.

25. Stehr-Green P, Tull P, Stellfeld M, Mortenson PB, Simpson D. Autism and thimerosal-containing vaccines: Lack of consistent evidence for an association. Am J Prev Med 2003;25:101-6.

26. Santarelli L, Bracii M, Moccehiani E. In vitro and in vivo effects of mercuric chloride on thymic endocrine activity, NK and NKT cell cytotoxicity, cytokine profiles (IL-2, IFN- $\gamma$, IL-6); role of the nitric oxide-L-arginine pathway. Int Immunopathol 2006;6:376-89.

27. Grandjean P, Andersen EW, Budtz-Jergensen E, Nielsen F, Molbak $\mathrm{K}$, Weihe P, Heilmann C. Serum vaccine antibody concentrations in children exposed to perfluorinated compounds. JAMA 2012;307:391-7.

28. Corsini E, Sangiovanni E, Galbiati V, Viviani B, Marinovich M, Galli CL, Dell'Agli M, Germolec DR. In vitro characterization of the immunotoxic potential of several perfluorinated compounds (PFC). Toxicol App Pharmacol 2012;258:248-55.

29. Dong GH, Zhang YH, Zheng L, Liu W, Jin YH, He QC. Chronic effects of perfluorooctanesulphonate exposure on immunotoxicity in adult male C57BL/6 mice. Arch Toxicol 2009;83:805-15.

30. Vetvicka V, Vetvickova J. Effects of glucan on immunosuppressive actions of mercury. J Med Food 2009;12:1098-104.

31. Vetvicka V, Vetvickova J. Glucan-resveratrol-vitamin C combination offers protection against toxic agents. Toxins 2012;4:1301-8.

32. Vetvicka V, Vetvickova J. Reversal of perfluorooctanesulfonate-induced immunotoxicity by a glucan-resveratrol-vitamin $\mathrm{C}$ combination. Orient Pharm Exp Med 2013;13:77-84.

33. Bayindir T, Filiz A, Iraz M, Kaya S, Tan M, Kalcoiglu MT Evaluation of the protective effect of beta glucan on amikacin ototoxicity using distortion product otoacoustic emission measurements in rats. Clin Exp Otorhinolaryngol 2013;6:1-6.

34. Pourahmad J, Ghashang M, Ettehadi HA, Ghalandari R. A search for cellular and molecular mechanisms involved in depleted uranium (DU) toxicity. Environ Toxicol 2006;21:349-54.

35. Shaki F, Pourahmad J. Mitochondrial toxicity of depleted uranium: Protection by beta-glucan. Iranian J Pharmaceut Res 2013;12:131 40.

36. Vetvicka V, Vashishta A, Saraswat-Ohri S, Vetvickova J. Immunological effects of yeast- and mushroom-derived pglucans. J Med Food 2008;11:615-22.

37. da Silva AF, Sartori D, Macedo FC, Fungaro MHP, Mantovani MS Effects of $\beta$-glucan extracted from Agaricus blazei on the expression of ERCC5, CASP9, and CYPIAI genes and metabolic profile in HEPG2 cells. Human Exp Toxicol 2013;32:647-54. 